\title{
RED AND/OR INTERESTING AT MSA '93
}

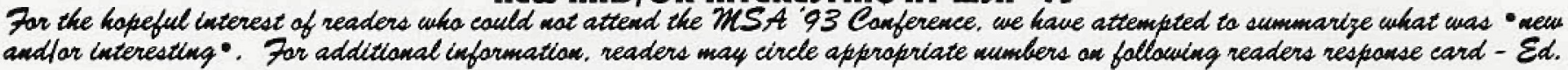

(1) 4pi Analysis introduces Macintosh-based, High-Performance Digital Image Acquisition for SEMISTEMs (analog and digital). The system utilizes existing imaging programs (NIH Image, IPLAB Spectrum, Adobe Photoshop). Also available: EDS spectral acquisition using NIST/NIH DTSA. 4pi Analysis: (919)489-1757. Fax: (919)489-1487

(2) AMRAY, INC. displayed the Model 1910 FE SEM with an integrated Link ISIS System. One PC controls all SEM and EDX functions working off a Windows platform. AMRAY, INC: (800)225-1462 or (617)275-1400. Fax: $(617) 275-0740$.

(3) On display were the Kimball Physics ES-423E Extended Life LaBs Cathodes which combines easy operation with exceptional thermal, chemical, mechanical and electrical stability. Also on display were Tungsten filaments, nonevaporable Geller Pumps, sputter \& carbon coaters and other EM accessories. The exclusive North American distributor for retail sales of Kimball Physics: BARRY SCIENTIFIC, INC:: $(800) 348-2257$. Fax: (508)347-8280.

(4) A new Electron-Beam Style Bench-Top Coating System for producing ultra thin carbon support films, which have applications in very high resolution studies using STEM and HVTEM. The films can routinely be produced and measured to thicknesses between 1.5 to $2.0 \mathrm{~nm}$, or greater if necessary. Cressington Scientific Instruments: (412)772-0220. Fax: (412)772-0219.

(5) Our quantitative $x$-ray microanalysis systems, Micro.EDS and MicroPlus now include Standardless Analysis. The newly introduced Macintosh based MicroMate, for $\$ 6,500.00$, provides EDS acquisition, all the display features of MicroPlus, and ASCII data files in DTSA and EMSA formats. Dapple Systems: (408)733-3283. Fax: $(408) 736-2350$

(6) Another MSA Meeting with presentations of the PS1000 Metal-Mirror Cryofixation Device. Quality cryofixation that fits everyone's budget. Call Delaware Diamond Knives for references and to discuss your application: (800)222-5143. Fax: (302)999-8320.

(7) DIATOME had on display the new "Static-Line" II for perfect ribbons of cryo sections, their Diamond Trimming Tools for ambient and cryo sectioning, as well as their complete line of Diamond Knives $(35,45,55$ degree angles - ultra-thin, semithin, cryo-wet, cryo-dry, histo-thick) for sectioning biological and material specimens DIATOME U.S.: (215)646-1478. Fax: ((215)646-8931.

(8) EDAX International released a number of new products at the MSA Meeting The new DX-5NT is the world's first EDS system incorporating Pentium technology and Windows NT. Also new from EDAX, DX-LIVE, for the fastest $X$-ray mapping available. DX-LIVE provides $X$-ray maps in a few seconds. Another new product presented by EDAX is DX-AUTO for fully automated EDS analysis including image drift correction. Detector performance has been improved with the introduction of the latest $P^{2}$ A pulse processor. For further information on these exciting products, contact EDAX INTERNATIONAL: (201)529-4880. Fax: (201)529-3156.

(9) Egoltronics showed their EM-55 Scanning Enlarger System for photographically enhanced reproduction of EM negatives. The EM-55 features automatic contrast control with electronic "dodging and burning" to reveal detail lost in conventional reproduction. Also shown was the TEK 14, one of their full line of paper and film processors. EGOLTRONICS: (703)335-1501. Fax: (703)335-1234.

(10) Many new innovative microscopy products were on display including the precision pulsed Laboratory Microwave Ooven, the semi-automatic Oscillating Tissue Slicer, the chromium Sputter Coater, the complete line of aurion immunogold reagents and accessories, and all of the chemicals, supplies, accessories, and equipment for the entire microscopy field. Electron Microscopy Sciences: (215)646-1566. Fax:: (215)646-8931.

(11) ETP USA introduced its new infrared CHAMBER VIEW SYSTEM at MSA The ETP system features POWER BURST illumination and at $\$ 2,950$ also includes a $9^{\prime \prime}$ monochrome monitor. Also shown was the new microprocessor controlled SERIES 4 Robinson Detector featuring LED displayed detector status. ETP USA: (800)8 ETP USA. Fax: $(510) 449-8996$

(12) M.E. Taylor Engineering, Inc. offers FORELINE TRAPS to keep oil vapors in the roughing pump from migrating to the specimen chamber, diffusion or turbo pumps, and $\mathrm{X}$-ray detectors. Installs in roughing pump hose line. Trapping material (elements) easily replaced at low cost. M.E. Taylor Engineering, Inc.: (301)774 6246. Fax: (301)774-6711
(13) NORAN Instruments demonstrated the microscopy lab of the future fealuring VOYAGER, the workstation-based microanalysis system. Two VOYAGERS on electron microscopes were networked with an ODYSSEY Confocal Microscope, Pentium PC, Macintosh, and Sun workstation, as well as color and black and white printers, demonstrating the power of sharing information in the laboratory and throughout the world. NORAN Instruments: (608)836-4330. Fax: (608)831-2313.

(14) OPTICON's new, all stainless steel, all-reflective Microscope Objectives are available in $15 X$ magnification, to complement the $15 X$ and $36 \mathrm{X}$ standard reflective objectives, OPTICON's objectives offer long working distances, figh numerical apertures, freedom from chromatic aberrations, and functionality in the UV through the infrared. Special coatings are available to suit varying applications. OPTICON: (508)663-6105. Fax: (508)663-0015.

(15) Optronics Engineering announces the TEC-470 thermoelectrically cooled CCD Color Camera that produces extremely sharp, clear video images when integrating up to four minutes. At $35^{\circ} \mathrm{C}$ below ambient temperature, true color display of images that exceeed human vision are visible. This camera has superior resolution and a signal-to-noise ratio of 53db. The electronic auto exposure mode compensates for fluxuations in illumination from 13,000 - 0.0002 LUX. Optronics Engineering: (805)968-3568. Fax: (805)968-2046.

(16) New producis from the Oxford Microanalysis Group were the Link GEM High Resolution Detector guaranteeing $115 \mathrm{eV}$ at Manganese, $65 \mathrm{eV}$ at Fluorine and 133 $\mathrm{eV}$ at 10,000 CPS acquisition rate, plus IMQuant, an image analysis package for the Link ISIS X-ray microanalysis system that includes both chemical and morphological typing. OXFORD MICROANALYSIS GROUP (U.S.): (508)369-8850. Fax: (508)3698287 .

(17) Peak Instruments used the recent MSA forum to introduce its FOCUS PIus WD X-Ray Spectrometer, which builds on the industry success of the Focus MCS-4. Incorporating Peak's unique "Positioning by Independent Parameterization" (PIP) technology for enhanced speed and repeatability, the easy to interface FOCUS PIUS also expands the range of elemental coverage by offering 4,5 or 6 crystals. A new software package, available unders Windows or UNIX, stand-alone or integrated into your ED system, completes the product. For your tough, repetitive WDS analysis needs, the application-tailored Focus Plus is the cost effective solution. Peak Instruments: (609)737-8133. Fax: (609)737-1724.

(18) PGT ushered in a new era in X-ray microanalysis with the untroduction of PRISM, the first EDS detector with digital pulse processing. This landmark development yields the highest $X$-ray throughput in the industry with substantial improvements in sensitivity, precision and total detection effieiency for all elements. The ability to process more of the incoming $X$-ray photons with less system dead time makes PRISM particulary advantageous for use with field emission SEMs, beam sensitive samples and other low beam current analyses. For information on PRISM and the 2nd generation, SUW workstation based IMIX-XD analyzer, contact Princeton Gamma-Tech: (609)9247310. Fax: (609)9241729.

(19) Queensgate Insiruments demonstrated their System 2000 product line that enables scanning microscopes to measure and position samples to $0.1 \mathrm{~nm}$ Combining ultrasensitive capacitance micrometers via servocircuits to multiaxis piezoelectric transistor stages allows precision $10 \mathrm{X}$ better than all other techniques QUEENSGATE INSTRUMENTS, INC.: (516)623-9725. Fax:(516)546-7031.

(20) in addition to their computerized stages and custom tailored SEM accessories, Raith introduced Proxywriter: an enhanced replacement for ELPHY-I, the University version of their SEM lithography system. ELPHY PLUS, a powerful Windows based lithography package, was also on display for the first time. This enhanced system, combined with Raith's laser interferometer stage and high speed electrostatic beam blanker, allows low volume R\&D labs "in house" mask making and direct write capabilities at a fraction of the cost of a full production system. RAITH USA, INC.: (516)293-0870. Fax: (516)293-0187.

(21) SPI SUPPLIES introduced for the first time ever its new line of Precision Plastic Tweezers, including ESD compatible formulations. Use in environments where no metal ions can be tolerated. Won't corrode and tips won't break if dropped. Significantly cheaper than metal. Contact SPI Supplies for descriptive price sheet (800)2424SPI or (215)436-5400. FAX: (215)436-5755. 
(22) Topcon Technologies, Inc. is proud to announce a new 10-year warranty that is included with their complete line of scanning electron microscopes (SEMs). Topcon Technologies, Inc. sells and services Topcon SEMs in the United States. The Topcon approach to SEM design emphasizes value, versaility, and ease of use. Tungsten, $\mathrm{LaB}$, and field emission instruments are available, and all Topcon SEMs can be operated under Windows control or from the standard operator's console. Instruments can be configured with a 2048 pixel imaging-processing system and low vacuum operating capability and have many other useful accessories. Topcon Technologies: (800)538-6850. Fax: (415)462-2234.

(23) TopoMetrix made it clear that they are not only continuing their aggressive marketing program, but also they are investing in a big way in new technology. An example was their announcement of the first commercially available scanning nearfield optical microscope (SNOM) - . - they call it Aurora - . - scheduled for shipment in the third quarter. TopoMetrix: (408)982-9700, Fax: (408)982-9751.

(24) DIFFRACTION WORK MADE FUN AND EASY?! Desktop Microscopist Diffraction Simulation and Analysis Software for the Macintosh can provide up to $10 x$ savings in the time required for typical TEM diffraction analysis. Call Virtual Laboratories for a free demo disk: (707)462-8037. Fax: (707)462-5275.

(25) Vital Image Technology displayed the new Seikosha VP-4500. A true 8-bit printer that accepts $10-135 \mathrm{MHz}$ video signals. VIT also introduced the Mitsubishi CP-1000, a color dye-sub printer that replaces the CP-210. For information regarding these printers, networkable printers or consumables for printers, call Vital Image Technology: (800)860-4624, Fax: (805)297-3814.

(26) The Carl Zeiss EM912/OMEGA TEM was exhibited officially for the first time at MSA-Cincinnati. This TEM, which utilizes an integrated, self-compensating, image spectrometer, had previously been selected to receive a prestigious R\&D 100 award. Several different papers involving novel electron spectroscopic diffraction andior imaging applications were presented at the meeting. CARL ZEISS, INC.: (800)356-1090. Fax: (914)681-7443.

\section{Diffraction \\ Simulation and Analysis Software}

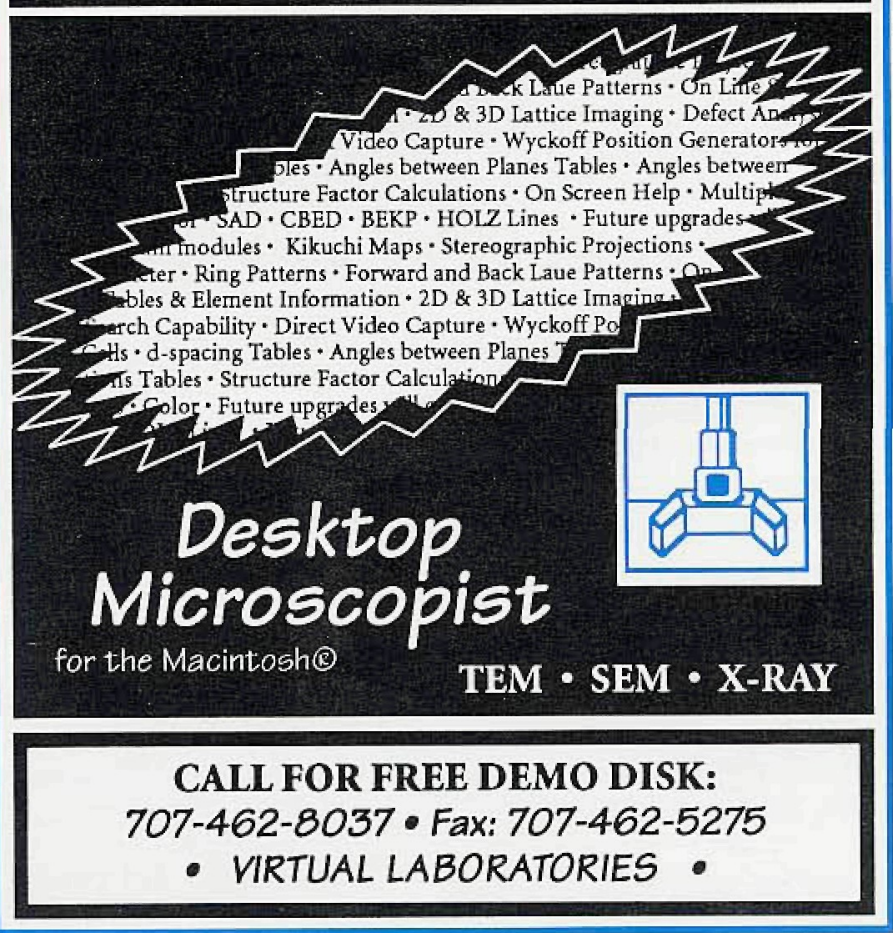

FROM:

$\mathrm{Dr}, \mathrm{Ms}$ _ Mr

Company:

Dept/MS/BIdg/etc.

Street

City State___ Zip

Telephone Number

Please provide me with further information on the products/services that I have checked below:

A EM912/OMEGA TEM: Carl Zeiss, Inc.

B NanoScope - MultiMode SPM: Digital Instruments

C 2020 Enviromental SEM: Electroscan

D X-Ray Detectors: GDE, INC.

E PC-Based Digital Image Acquisition System: HNU Systems.

F AFM \& STM by Leica \& WYKO: Leica, Inc.

$G$ EMC Magnetic Field Compensation System: Linear Research Associates.

H SEM Scintillators \& Foreline Traps: M.E. Taylor Engineering, Inc.

I Customized On-Site Training: Microscopy \& Microscopy Training.

J SPM - AutoProbe CP: Park Scientific Instruments.

K SPM - Experimenter's Tool Kit: TopoMetrix

L Diffraction \& Simulation Software: Virtual Laboratories

And, from "New And/Or Interesting at MSA '93", on the products whose preceding numbers I have circled below:

$\begin{array}{llllllll}1 & 2 & 3 & 4 & 5 & 6 & 7 & 8 \\ 9 & 10 & 11 & 12 & 13 & 14 & 15 & 16 \\ 17 & 18 & 19 & 20 & 21 & 22 & 23 & 24\end{array}$

19
23

Readers who wish to continue to reciue a no cost cops of this meusletter and who have previously not dame saare eneouraged to complete the following. Thank you!

We would properly be considered a (check the most appropriate): A User of microscopy equipment, materials or services.

B Supplier of microscopy equipment, materials or services.

Our interests include the (check all applicable):

C Physical/Material Sciences.

D Biological/Biomedical Sciences.

E Earth Sciences.

My own professional interests include (check all applicable):

F Electron Microscopy

G Confocal Microscopy

H SPM/AFM

I Light Microscopy

J IR Microscopy

K Acoustic Microscopy

After completion, please detach along "perf". fold and tab elased with sesteh tap. Thande you!
18
26

25 
microscopy through the use of annular dark field microscopy, an approach that is direct (i.e, does not involve the intermediate computational steps). It permits the use of forwardly scattered electrons for electron energy analysis. Again, new insight into the local chemistry, bonding and electronic structure is becoming available in parallel with the image acquisition Finally, the correction of the aberrations of electron lenses, a longstanding problem, is being tackled again in Germany with an approach that offers real prospects of success. If this succeeds, the the way to 0.5 Angstrom resolution appears open. Further success with this approach is likely eventually to make the construction stages for in-situ atomic structure studies much easier than at the present time.

Progress in thes areas and others were reviewed in a panel discussion at the Microscopy Society of American annual meeting in Cincinnati, Ohio this August. The updates provided at this meeting confirmed that progress since the May workshop had been as expected and that these new tools are becoming available for use as projected.

In its report to the NSF, "Atomic Imaging and Manipulation for Advanced Materials", the NSF panel on Atomic Resolution Microscopy identified areas of both scientific and technological significance where these approaches could make significant contributions to many scientific disciplines and to U.S. national priorities. It concluded that a strong case existed for ensuring continued U.S. participation in these areas at an internationally competitive level and recommended NSF annual support levels of $\$ 20$ million per year over the next five years. The report is expected to become generally available in early September, 1993.

\section{USED EOUIPMEMT FOR SALE}

$\checkmark$ Philips EM 400T TEM with Tracor Northern (Noran Instruments) TN-5400 EDS System and Haskris water chiller. Under continous Philips service contract until this year. Currently in use. Contact Jimmy Neal, Alabama Power Company: Tel.: (205)250-1773, Fax: (205)250-1902

$\checkmark$ Two ARL SEMQ Electron Microprobes. SN 45 and 74. Reconditioned like new. Four WDS scanning spectrometers, 52.5 degree takeoff. GW Electronics BSE system. Kevex EDS detector. Updated with our Micro-3WD automation system which includes WDS, EDS, Digital Imaging, Image Analysis, and high speed optical servo motors. Includes 1 year warranty. $\$ 150,000$ each. Call for demo disks and more information. Advanced MicroBeam Inc. Tel.: (216)-394-1255, Fax: (216)394-1834.

$\checkmark$ EDAX PV9800 Energy Dispersive X-ray Microanalysis System, EDAX Detector (New 1992), EDAX Monitor (New 1992), EDAX OPCON Keyboard, HP Thinkjet Printer, Bio-Rad Plasma Asher, Edwards E306A Carbon Coater and Edwards $5 \mathrm{HP}$ vacuum pump. Equipment currently in use and in excellent condition. For more information, contact Karen Green: Tel.: (205)922-0304

$\checkmark$ Two HITACHI TEMs, HU-11E and HU-11C, available immediately for cost of moving. Both operative. Well cared for. Ideal for teaching. HU-11E located at the University of Florida in Gainesville, FL. Contact Dr. Henry C. Aldrich at tel. (904)392-1096. HU-11C located at Eckerd College in St. Petersburg, FL. Contact Dr. Sheila Hanes at tel. (813)864-8532.

$\checkmark$ HITACHI S-2400 SEM, 2 years old. With Kevex Level 1 EDS system (including X-ray mode and Superquantum, and Quantum X-ray detector), Robinson BSE detector, raster ratation, graphics generator,

\section{USED EOUIPMEAT UUATED}

$\checkmark$ Used transmitted and/or reflective polarized light microscope with rotating stage and tinocular head. Material Characterization Services, Sue Pignolet Brandon: Tel.: (708)548-6522
Setting the Pace in Microscopy...

\section{...through quality, innovation and customer support.}

Carl Zeiss, Inc. offers a wide range of pace setting products, featuring the latest innovations in microscopy.

- EM 912 OMEGA TEM - newest TEM with innovative, leading edge technology - OMEGA electron energy spectrometer

- EM 910 TEM - versatile TEM providing advanced Koehler illumination

- EM 902 TEM - trendsetting, economical TEM with integrated electronic energy spectrometer

- DSM 940A - fully digital, economical, SEM with superb value and features

- DSM 960A - large chamber SEM offering high performance and versatility

- DSM 962 - research grade SEM featuring superior high-resolution image storage and processing

Keep up the pace of progress. Take advantage of Zeiss' first rate products and dependable customer service.

(800) 356-1090

Fax (914) 681-7443

Carl Zeiss, Inc.

Electron Optics Division One Zeiss Drive

Thomwood, New York 10594
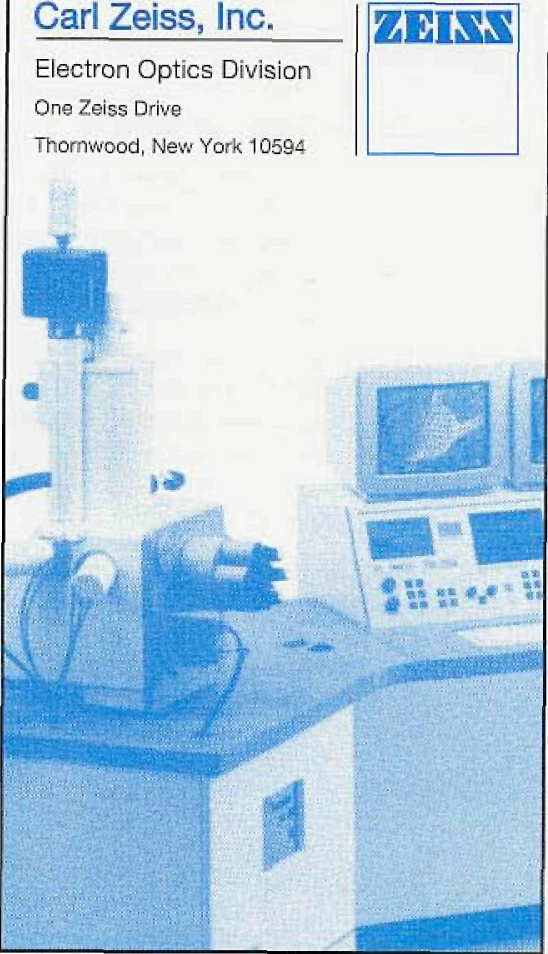


\section{Powerful Pairs for Challenging Applications}

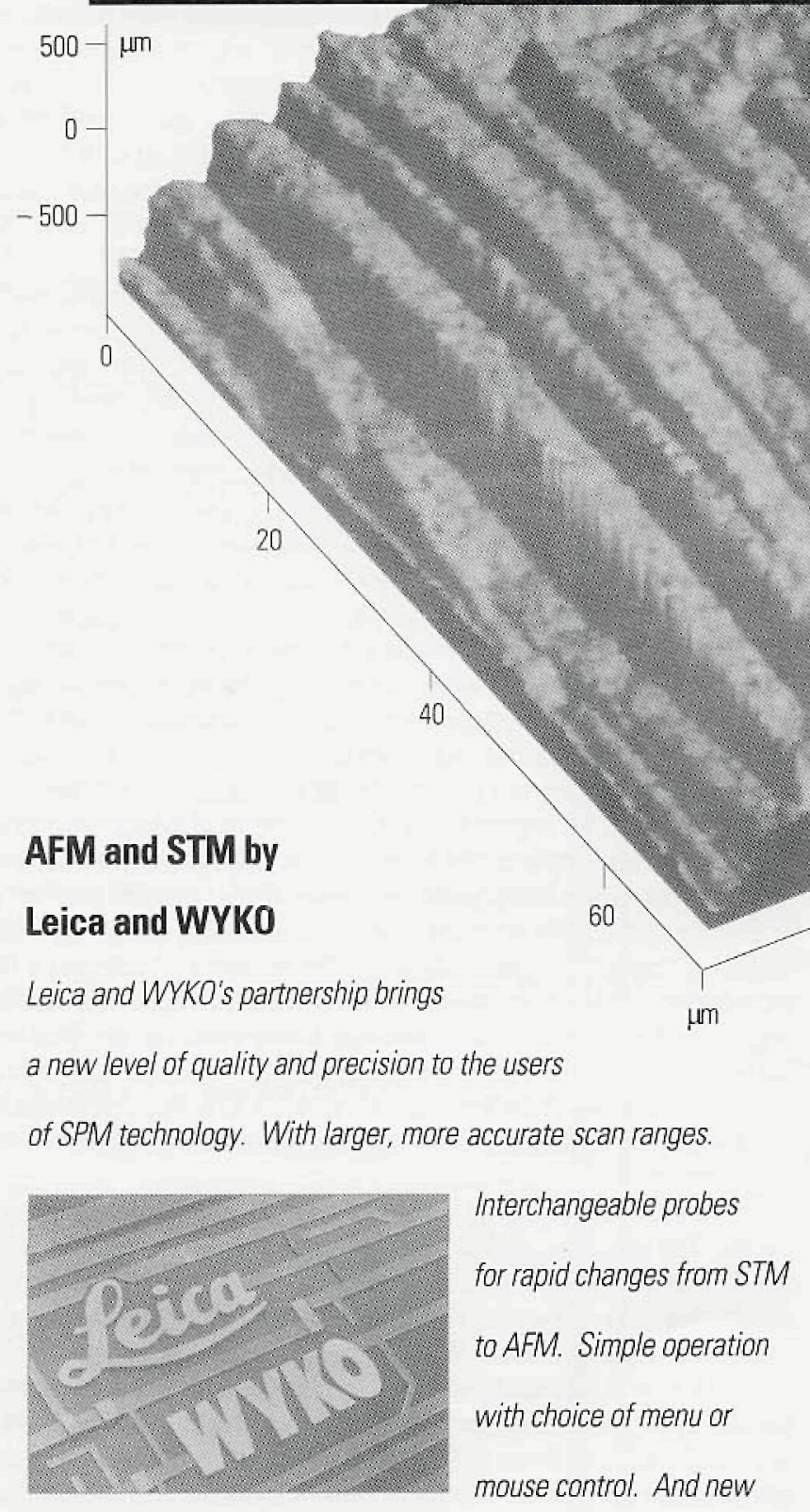

levels of product assistance and support.

Both the Model SPM 20 and SPM 30 give you accurate results quickly and easily. Both measure samples up to $30 \mathrm{~mm} \times 30 \mathrm{~mm} \times$ $20 \mathrm{~mm}$ thick or entire disks to $95 \mathrm{~mm}$ diameter. Both have an intuitive, graphical interface with powerful control and analysis software.

The SPM 30 features automated sample positioning and probe loading, advanced optical position encoder with closed-loop scanner, remote controlled side-view microscope with video display, and integrated enclosure and vibration isolation system.
Leica Canada inc.

513 McNicall Averue

Willowdale, Ontario $\mathrm{M} 2 \mathrm{H} 2 \mathrm{Cg}$

416/497-2460 FAX 416/497-2053
The heart of the SPM 30 is a patented, closed-loop scanner with optical position encoder to eliminate the negative effects of hysteresis, non-linearity, creep and drift. The bottom line is fast accurate dimensions in $X, Y$ and $Z$ !

The Leica/WYKO SPM 20 and SPM 30 offer you the best in today's SPM technology, backed by two companies that stand for quality, support and innovation. For more information and the name of the Leica office nearest you, call us at 1-800-248-0123

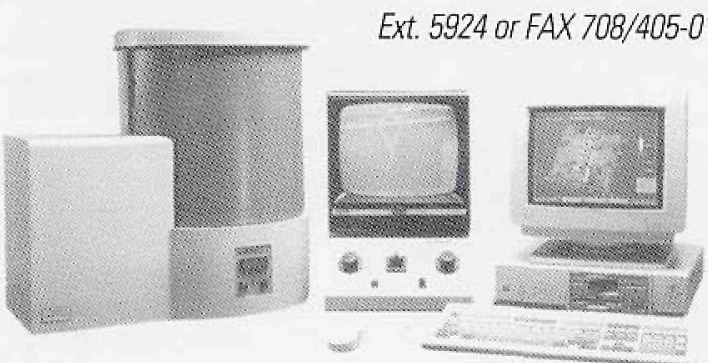
Ext. 5924 or FAX $708 / 405-0147$. 


\section{OOMIIG EVEnTS}

$(* *)$ : Contact Microscopy Today for further information.

$\checkmark$ Sept 8/10 '93: Microscopy/Photomicrography. American Type Culture Collection. Rockville, MD. Brian K. Wiggins: (301)231-5555.

$\checkmark$ Sept 17/19 '93: 12th Annual Symposium on Advances in Microscopy (Duke Univ/NC Society for EM). Pine Knoll Shores, NC. Betty Gooch: (919)684-3534

$\checkmark$ Sept 26 - Oct 2 '93: 2nd International Congres on Electron Microscopy. Cancun, Mexico. Mario Meki: Tel.: (525)622-50-33, Fax: (525)548-31-11.

$\checkmark$ Oct 577 '93: Third Annual Analytical Laboratory Exposition and Conference (ALEX '93). San Francisco, CA. (61\&)449-8938.

$\checkmark$ Oct 25/29 '93: Scanning Electron Microscopy Field Emission and X-Ray Microanalysis. (SUNY) Ellenville, NY. Dr. Angelos Patsis: (914)257-3800.

$\checkmark$ Nov 17/21 '93: Nat'I Assoc of Biology Teachers Convention. Boston. (703)471-1134

$\checkmark$ Nov 15/19 '93: 40th Annual Symposium of American Vacuum Society. Orlando, FL. Marion Churchill: (212)661-9404.

$\checkmark$ Nov 29/Dec 3'93: MRS Fall Meeting. Boston, MA: (412)367-3003.

$\checkmark$ Feb 27/March 4 '94: PITTCON '94. Chicago IL Alma Johnson (412)825-3220.

$\checkmark$ Mar 14/18 \& 21/25 '94: Practical Aspects of Scanning Electron Microscopy. (Univ. of MD 4.5 day short courses). College Park MD. Tim Maugel: (301)405-6898.

$\checkmark$ April 5/7 '94: MRS Spring Meeting. San Francisco CA. Mary E. KLaufold: (412)367-3036.

$\checkmark$ May 7/12 '94: Food Structure Annual Meeting. Toronto, Canada. Dr. Om Johari: (708)529-6677

$\checkmark$ May 17/20 '94: SCANNING '94. (FAMS \& SEEMS) Charleston SC. Mary K. Sullivan: (201)818-1010.

$\checkmark$ June 13/23 '94: Lehigh Microscopy Short Cources. Bethelem, PA. David B. Williams. (215)7585133.

d June 26/30 '94: 10th Annual Molecular Microspeciroscopy Short Course. (Miami University) Oxford, OH. (513)529-2873.

$\checkmark$ July 17/22 '94: 13th International Congress on Electron Microscopy. Paris, France. Secretariat ICEM 13,67 rue Maurice Gunsbourg, 94205 Ivry sur Seine cedex, France.

$\checkmark$ Sept 12/15 '94: MICRO 94 - International Microscopy and Image Analysis. London, UK ( $\left.{ }^{\star \star *}\right)$

\section{REGIONAL MSA/MAS EVENTS}

Oct 9 '93: Pacific Northwest EMS Meeting. Univ of Idaho, Moscow ID. Charlie Meshul: (503)220-8262.

$\checkmark$ Oct 14/14 '93: Louisiana SEM Meeting and Workshops. Tulane Univ., New Orleans LA. Owen P. Mills: (504)865-5142.

$\checkmark$ Oct 21/23 '93: Great Lakes Electron Microscopy Affiliates Conference. Airport Hilton Inn, Indianapolis IN. Sandy L. White: (317)737-6423.

\section{$\checkmark$ Oct 21/23 '93: Texas SEM Fall Meeting. Galveston, TX. Dr. Louis Bragg}

$\checkmark$ Oct 28/29 '93: Central States EMS Meeting and Cryomicrotomy Workshop. St. Louis Univ. School of Medicine, St. Louis MO. Dr. Jan Ryerse: (314)577-8480.

\section{Experimenter's Tool Kit}

SPM 1 , scanning Probe Microscopy, is the fastest Research that, by its very nature, involves experimentation. And experimentation demands flexible instruments.

To meet this important need for flexible SPM instruments, TopoMetrix offers the Experimenter's Tool Kit.

We're well known in the SPM community as the one company that offers "open-architecture" SPMs. Open-architecture electronics that permit direct, easy access to control and data functions. Open-architecture software - D-BOSS - a high-level instrument control language that lets you change experiment parameters and functions to suit your needs. And open-architecture hardware that accommodates a wide range of scanning heads and sample geometries.

Whether your need is electronics, software, hardware, or complete systems, TopoMetrix thinks you should buy what you want, when you want, and configure it the way you want.

To learn more, call us today at 1-800-765-5067. Then you can get started on an Experimenter's Tool Kit that's exactly right for you.

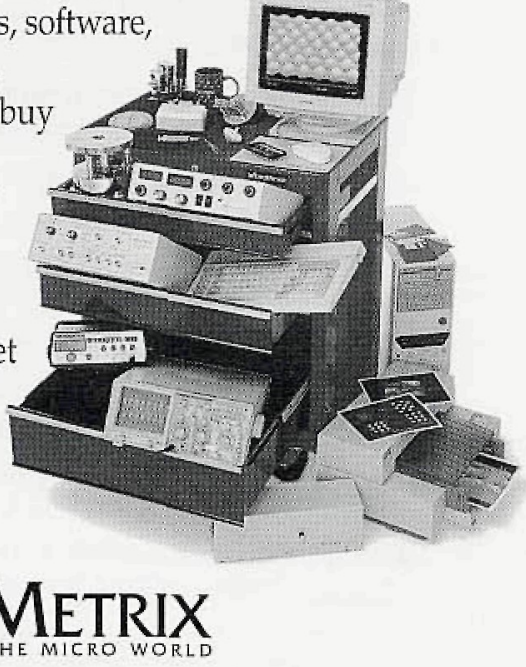

5403 Betsy Ross Dhive, Santa Clara, Californin 95054 TEL 408.982.9700 FAX 408.982 .9751 


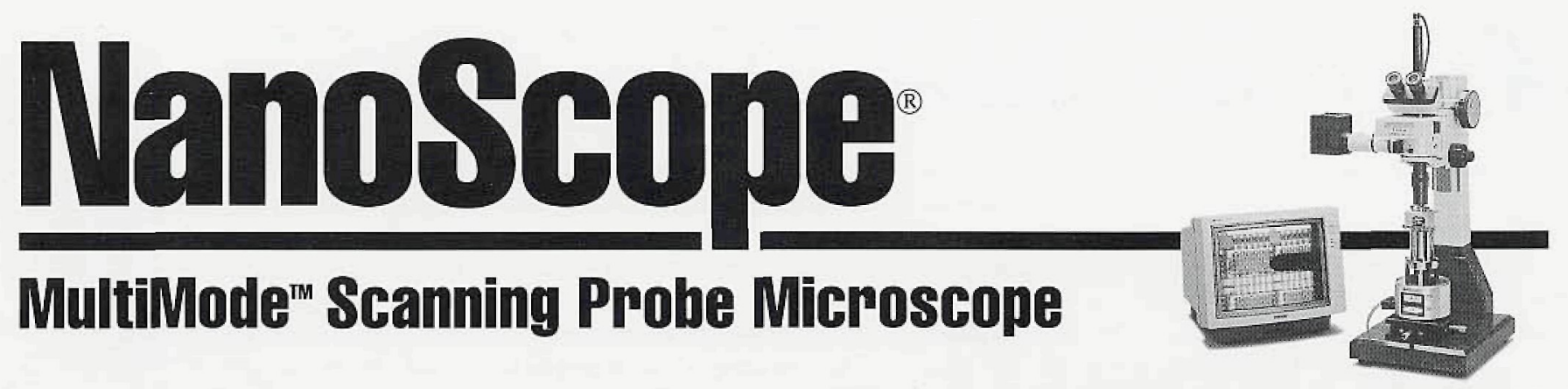

Magnetic Force Gradients D

These hard disk bits were written with alternating

polarity and a slight skew.

The speckle above and

below the recorded track

is due to the disordered

magnetic domains in the virgin media
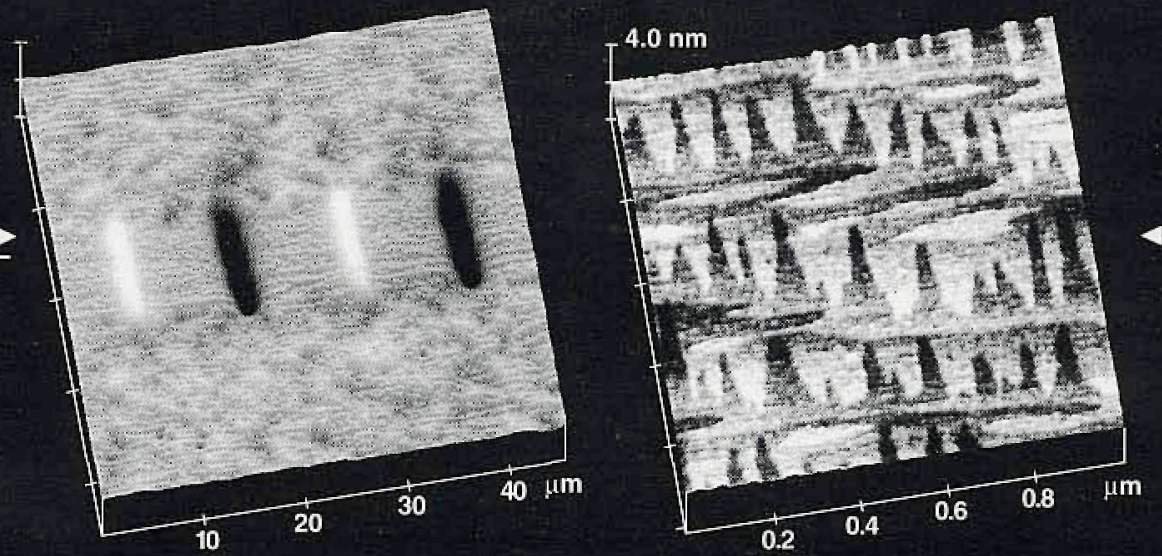

TappingModeTM

AFM Topography

These 1.6A-high terraces

of epitaxially-grown silicon

were imaged using the

NanoScope Large Sample

Stage. Only the AFM probe

touched the top surface of

the intact 8 in wafer.
Lateral Force (Friction) $>$

A mixture of EPDM and natural rubber scanned with a $\mathrm{Si}_{3} \mathrm{~N}_{4}$ tip shows regions of higher friction (lighter color) and lower friction (darker color). These regions probably correspond to the two different types of rubber.
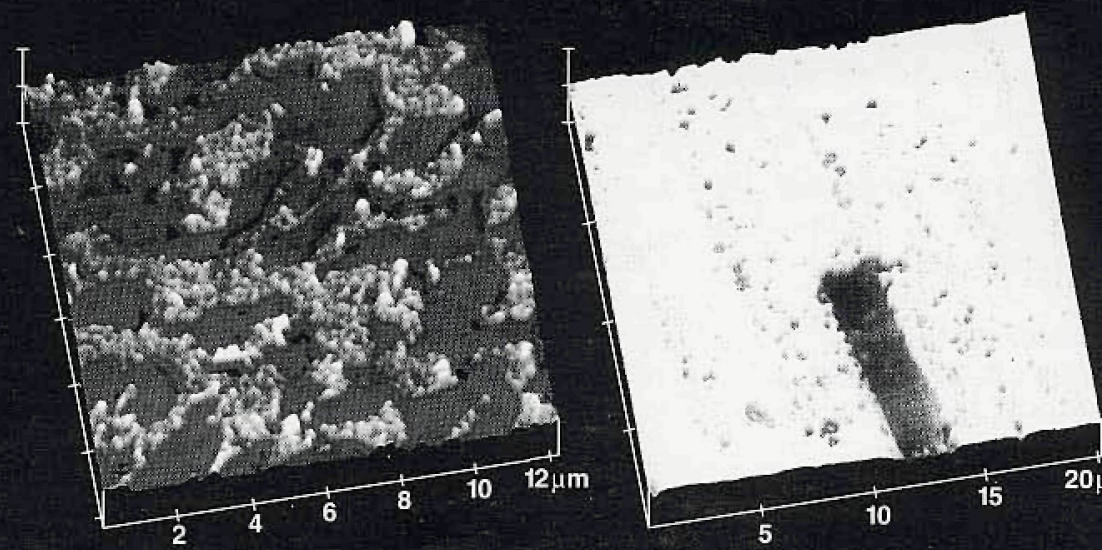

1 Electric Force Gradients A voltage applied to a broken metallization line on a GaAs test structure is shown. The image clearly indicates that the line is open at the break. This capability is another example of Digital Instruments innovation.

\section{Innovation and Performance...}

\section{Only The MultiMode SPM Can Make All These Measurements- And Do Them Routinely, Day In and Day Out}

$\mathrm{O}$

ver 1,000 users have chosen the NanoScope SPM because it provides them with the most powerful and innovative capabilities available. Our new MultiMode ${ }^{\mathrm{TM}}$ SPM is the next step in the evolution of the NanoScope system. It operates as a

TappingMode ${ }^{\mathrm{TM}}$ AFM for superior measurements on even the most difficult samples, and as a regular contact AFM, a noncontact AFM, an STM, a magnetic force microscope (using our exclusive magnetic NanoProbes), a lateral force microscope, and more capabilities are being added. The Electric Force image above is a glimpse of things to come- and yet another example of Digital Instruments' innovation in SPM technology.

Call today to discuss your application, and to find out what makes the NanoScope the most popular SPM system in the world. We deliver results, not promises.

\section{di Digital Instruments}

Santa Barbara, CA - TEL: 805-899-3380 or 800-873-9750 FAX: 805-899-3392

Japan: Toyo Corp. •TEL: 81-3-5688-6800 •FAX: 81-3-5688-6900 Other Distributors Worldwide 


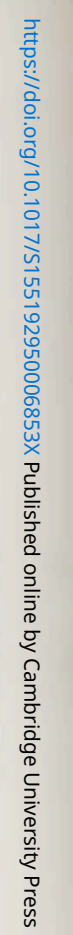

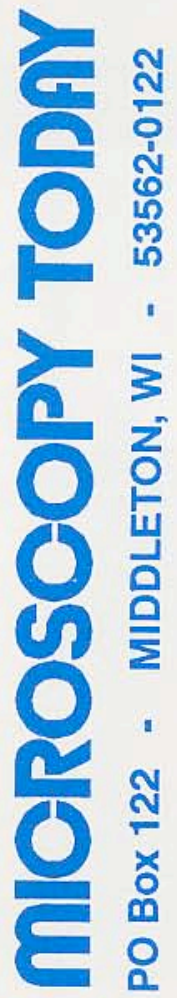




\section{A Berry Sweet Development: Microscopy of the Future Jean-Paul Revel, CALTECH}

One of the most pervasive problems in microscopy is that of making sure that what one is observing represents reality. The complex specimen preparation steps which have to be followed to obtain images of biological samples all produce artifacts, at least in the sense that they modify the sample. The investigator has to somehow show that these changes in the sample reveal true microscopic features, rather than structures which do not exist in the living. This, of course, is why polarized light microscopy, phase and interference microscopy and their derived approaches, all of which reveal detail in live untreated specimen, are so important, in spite of the limited resolution which can be achieved.

When electron microscopy was in its infancy there were, and justifiably so, even more severe fears about the reality of what could be seen. Even visionary people like Gabor had their doubts and the first steps in biological microscopy were taken with the realization that if the sample was burned to a crisp, perhaps examination of its cinders would still give valuable information. It was very important in the early days to have independent confirmation of the structures and this was to some extent achieved by comparison of the data obtained by $X$-ray diffraction experiments with that revealed by electron microscopy. Thus the work of pioneer biophysicists on nerve myelin and other "quasi" crystalline systems was of great importance. The myelin sheath of nerve was a regular array of membranes which could be studied "alive" by X-ray and also after processing for electron microscopy

At the MSA meeting in Cincinnati last month it became obvious even to this jaded microscopist that another approach to the study of live samples was likely to become increasingly important. The technique in question is nuclear magnetic resonance (NMR) imaging (see Wehrli ${ }^{2}$ for review), also called MRI so as not to raise fears associated with anything atomic. It is already a very powerful tool in the hands of the physician because insofar as one can presently ascertain it is "non invasive" and also gives good contrast in soft tissues. It is often used in imaging brain and other body structures. Even today it can be applied to the study of some dynamic events such as blood flow. As a result magnetic resonance angiography is a reality. Spectroscopic imaging, a very promising approach, allowing metabolic studies in vivo, is also being used for medical and other purposes. An example of the increasing pervasiveness of MRI can be found in its application to the study of water, lipid and carbohydrate distribution in the

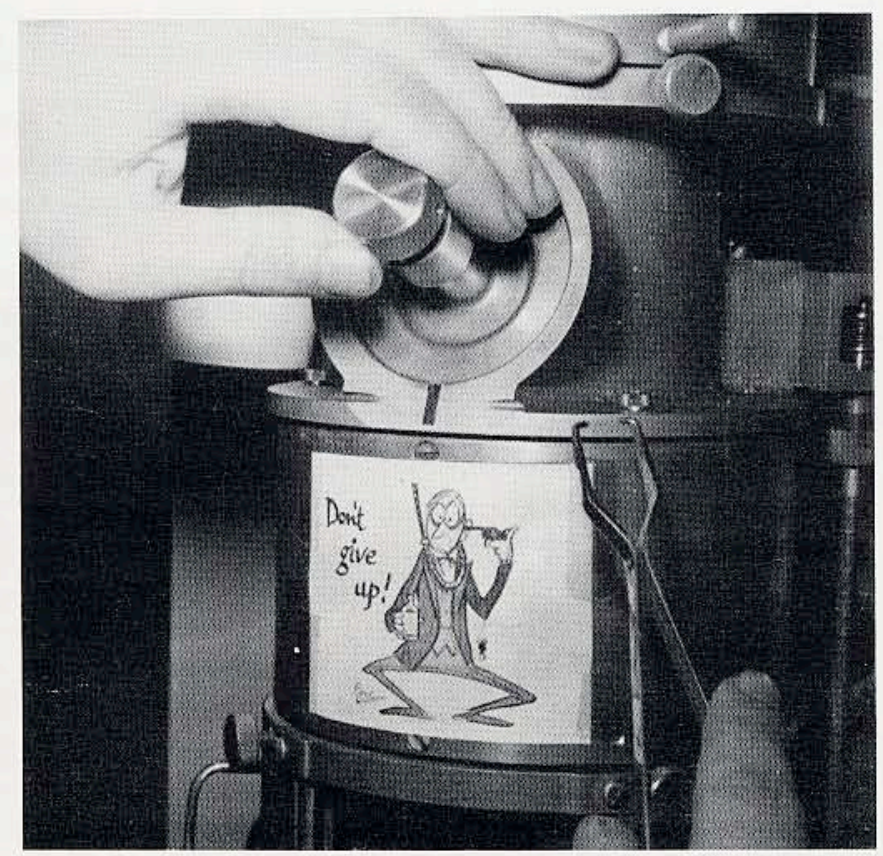

berries of Suitana, Chardonnay, Riesling and Shiraz grapes ${ }^{3}$, a berry sweet application indeed! NMR is also fast becoming a very important tool complementing $\mathrm{X}$-ray techniques, for the study of molecules in solution.

The results presented at the meeting showed that NMR is also about to invade, nay has already done so, the field of microscopy! J. Slides of the University of Washington in Seattle discussed new methods for the detection of magnetic resonance, derived in part on approaches to magnetic force microscopy. They measure the small oscillatory magnetic force $\left(10^{-14} \mathrm{~N}\right)$ acting on a paramagnetic sample (a smidgen $<30 \mathrm{ng}$ of diphenylpicrylhydrazide) which has been excited into magnetic resonance in an inhomogeneous magnetic field. The force is detected by optical sensing the vibration of a cantilever on which the sample is mounted ${ }^{4}$. While a spatial resolution of only 19 micron was demonstrated, it became clear that the prospects for the future are much greater than that. The audience at the Presidential Symposium where this data was presented was left breathless by the prospect of this elegant approach. Another presentation by R. Jacobs and S. Fraser from Caltech addressed the biological applications of microscopic MRI, showing scans of a mouse embryo in utero. This group has already shown several years ago that NMR microscopy could be used to image and follow individual cells in developing Xenopus embryo. Many problems have to be overcome if spatial resolution is to read in the submicron range, which theory says is possible. It appears however that ground is being covered rapidly and that MRI might well become one of the exciting new microscopies of the 21 st Century.

\section{References}

1) Marton L., Early History of the Electron Microscope. San Francisco Press, Inc 1968

2) Wehrli F.W. (1992) Physics Today, 45, 34-42

3) Pope J.M., Jonas D. and Walker R.R. (1993) Protoplasma, 173, 177-186

4) Rugar D., Yannoni C.S., and Sidles J.A. (1992) Nature 360, 563-566)

5) Cho Z, Ahn C., Juh S., Jo J., Friedenberg R., Fraser S., Jacobs R. (1990) Phil Trans. R. Soc. Lond. A $333,469-475$

\section{Save Thousands of Dollars and Eliminate Film in the SEM lab}

with the HNU DRV-2000

PC-Based Digital Image Acquisition System

Powerful

Collects up to six images in a single scan.

Fast Produces high resolution x-ray maps in minutes.

Versatile compatible with any scanning electron microscope.

State of the Art Fastest simultaneous digital imaging/x-ray mapping system available.

Economical Save thousands of dollars in instant film costs.

Pays for itself in only a few months.

Send for a free brochure or call 1-800-724-5600 for more information.

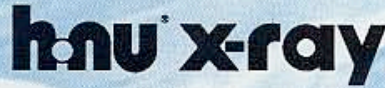

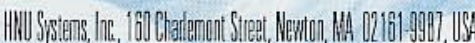

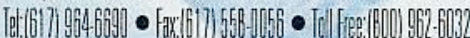

